\title{
Comportamento Viscoelástico Linear e Morfologia de Blendas PP/HDPE
}

\author{
Adriana M. C. de Souza, Nicole R. Demarquette \\ Departamento de Engenharia Metalúrgica e de Materiais, Escola Politécnica, USP
}

\begin{abstract}
Resumo: Neste trabalho, o efeito da composição da blenda PP/HDPE e da temperatura no comportamento viscoelástico linear e na morfologia destas blendas foi estudado. A tensão interfacial entre PP e HDPE foi avaliada utilizando o espectro de relaxação das blendas, seguindo as análises de Gramespacher e Meissner ${ }^{[1]}$. Os resultados indicaram que existe uma faixa de composições da blenda PP/HDPE para a qual é possível utilizar as análises de Gramespacher e Meissner ${ }^{[1]}$ na obtenção da tensão interfacial entre polímeros utilizando-se medidas reológicas. A compatibilização da blenda PP/HDPE também foi estudada através de análises morfológicas e da tensão interfacial entre os seus componentes. Três compatibilizantes foram testados: EPDM, SEBS e EVA. Curvas de emulsão relacionando o raio médio da fase dispersa e a tensão interfacial com a concentração de compatibilizante adicionado à blenda foram obtidas. Foi mostrado que o EPDM foi mais eficiente como agente emulsificante para a blenda PP/HDPE que o EVA e o SEBS.
\end{abstract}

Palavras-chave: Blendas, poliolefinas, tensão interfacial, reologia, compatibilização.

\section{Linear Viscoelastic Behavior and Morphology of PP/HDPE Blends}

\begin{abstract}
In this paper the influence of composition and temperature on the linear viscoelastic behavior and morphology of PP/HDPE blends was studied. The interfacial tension between PP and HDPE was evaluated using the relaxation spectra of PP/HDPE blends, following Gramespacher and Meissner analysis ${ }^{[1]}$. The results seemed to indicate that there is a range of compositions for which it is possible to use Gramespacher and Meissner analysis in order to calculate interfacial tension between polymers using small amplitude oscillatory shear measurements. The compatibilization of PP/HDPE blend was also studied through morphological analysis and interfacial tension between its components. Three types of compatibilizers were tested: EPDM, EVA and SEBS. Emulsion curves relating the average radius of the dispersed phase and the interfacial tension to the concentration of compatibilizer added to the blend were obtained. It was shown that EPDM was more efficient as an emulsifier for PP/HDPE blend than EVA or SEBS.
\end{abstract}

Keywords: Blends, polyolefins, interfacial tension, rheology, compatibilization.

\section{Introdução}

Devido ao seu relativo baixo custo e às suas propriedades versáteis, as poliolefinas são um dos termoplásticos mais utilizados ${ }^{[2]}$. Consequentemente, suas blendas têm atraído muito interesse ${ }^{[2-5]}$ Processabilidade e aumento nas propriedades de impacto do polipropilieno (PP) são razões suficientes para preparação destas blendas. Materiais dúcteis como polietileno ou elastômeros podem ser usados para este propósito. No entanto, o polipropileno (PP) e o polietileno (PE) são polímeros imiscíveis termodinamicamente, resultando numa blenda com fraca adesão entre as fases. Por essa razão, as blendas de polipropileno/polietileno (PP/PE) necessitam da adição de um agente compatibilizante para obter uma melhor adesão entre os componentes ${ }^{[3-5]}$. A eficiência da compatibilização pode ser avaliada através de curvas de emulsão introduzidas por Favis ${ }^{[6]}$. Esta curva relaciona o tamanho da fase dispersa (no caso de

Autor para correspondência: Nicole R. Demarquette, Departamento de Engenharia Metalúrgica de Materiais, Escola Politécnica da Universidade de São Paulo, Av. Prof. Mello Moraes, 2463, Cidade Universitária, CEP: 05508-900, São Paulo, SP. E-mail: amcs@usp.br/nick@usp.br 
uma morfologia de dispersão de gotas) com a concentração de compatibilizante adicionado à blenda. A eficiência da compatibilização também pode ser avaliada através de medidas de tensão interfacial entre os componentes da blenda. Dentre os vários métodos de medidas de tensão interfacial, poucos são utilizados para polímeros devido a sua alta viscosidade. Recentemente, vários trabalhos têm sido realizados com o objetivo de relacionar as propriedades viscoelásticas e a morfologia de blendas poliméricas ${ }^{[1,7]}$.

Alguns modelos teóricos têm sido desenvolvidos com o objetivo de se determinar o comportamento viscoelástico linear das blendas poliméricas sob fluxo. Estes modelos relacionam a resposta dinâmica das blendas poliméricas com sua morfologia, composição, e tensão interfacial entre seus componentes. É possível, portanto, utilizando estes modelos, obterse a tensão interfacial entre os componentes de uma blenda desde que a morfologia desta blenda seja caracterizada ${ }^{[1,7]}$.

Com o objetivo de avaliar a tensão interfacial entre polímeros através da análise do comportamento viscoelástico linear da blenda formada por estes polímeros, Gramespacher e Meissner ${ }^{[1]}$ desenvolveram uma equação constitutiva para uma emulsão baseada no trabalho de Choi e Showalter ${ }^{[8]}$ e em uma regra de mistura linear. Esta equação constitutiva considera o módulo complexo da blenda como uma combinação da contribuição das fases mais uma contribuição da interface. Estes pesquisadores obtiveram as seguintes equações para os módulos de armazenamento e de perda para uma blenda quando submetida a um ensaio de cisalhamento oscilatório de pequenas amplitudes:

$$
\begin{aligned}
& G^{\prime}(\omega)=\phi G_{d}^{\prime}(\omega)+(1-\phi) G_{m}^{\prime}(\omega)+\frac{\eta}{\tau_{1}}\left(1-\frac{\tau_{2}}{\tau_{1}}\right) \frac{\omega^{2} \tau_{1}}{1+\omega^{2} \tau_{1}{ }^{2}} \\
& G^{\prime \prime}(\omega)=\phi G^{\prime \prime}{ }_{d}(\omega)+(1-\phi) G_{m}^{\prime \prime}(\omega)+\frac{\eta}{\tau_{1}}\left(1-\frac{\tau_{2}}{\tau_{1}}\right) \frac{\omega \tau_{1}}{1+\omega^{2} \tau_{1}{ }^{2}}
\end{aligned}
$$

Sendo:

$$
\begin{aligned}
& \eta=\eta_{m}\left[1+\left(\frac{5 K+2}{2 K+2}\right) \phi+\left(\frac{5(5 K+2)^{2}}{8(K+1)^{2}}\right) \phi^{2}\right] \\
& \tau_{1}=\tau_{o}\left(1+\phi \frac{5(19 k+16)}{4(k+1)(2 k+3)}\right)
\end{aligned}
$$

$$
\begin{aligned}
& \tau_{2}=\tau_{o}\left(1+\phi \frac{3(19 K+16)}{4(K+1)(2 K+3)}\right) \\
& \tau_{o}=\left(\frac{\eta_{m} R}{\gamma}\right) \frac{(19 K+16)(2 K+3)}{40(K+1)}
\end{aligned}
$$

onde: $\mathrm{G}{ }_{\mathrm{d}}(\omega)=$ módulo de perda da fase dispersa; $\mathrm{G}_{\mathrm{d}}(\omega)=$ módulo de armazenamento da fase dispersa $\mathrm{G}^{\prime}{ }_{\mathrm{m}}(\omega)=$ módulo de perda da matriz; $\mathrm{G}_{\mathrm{m}}(\omega)=$ módulo de armazenamento da matriz; $\eta, \eta_{m}, \eta_{d}$ são as viscosidades newtonianas da blenda, matriz e fase dispersa respectivamente; $K=\left(\eta_{\mathrm{d}} \eta_{\mathrm{m}}\right)$; $\gamma$ é a tensão interfacial; $\phi$ é a fração volumétrica da fase dispersa e $\mathrm{R}_{\mathrm{n}}$ é o raio numérico médio da fase dispersa dado por:

$$
R_{n}=\frac{\sum_{i} n R_{i}}{\sum_{i} n_{i}}
$$

sendo $\mathrm{R}_{\mathrm{i}}$ o raio da i-ésima gota e $\mathrm{n}_{\mathrm{i}}$ o número de gotas de raio $R_{\mathrm{i}}$.

O termo $\tau_{1}$ corresponde a um tempo de relaxação extra no espectro de relaxação de uma blenda e está relacionado à relaxação da forma das gotas da fase dispersa, através do qual a tensão interfacial entre os componentes da blenda pode ser obtida.

Gramespacher e Meissner ${ }^{[1]}$ obtiveram a tensão interfacial através da análise do espectro de relaxação da blenda de poliestireno/poli(metacrilato de metila) (PS/PMMA) obtido por ensaios de cisalhamento oscilatório a $170^{\circ} \mathrm{C}$. Os resultados obtidos corroboraram com dados da literatura. Esse método de análise também foi utilizado por Gleinser et al. ${ }^{[9]}$ e por Macaúbas e Demarquette ${ }^{[10]}$.

Alguns estudos ${ }^{[11-14]}$ têm mostrado a possibilidade da influência da composição da blenda na determinação da tensão interfacial entre seus componentes utilizando-se o comportamento reológico desta blenda. No entanto, nenhum estudo sistemático foi realizado até o momento sobre o efeito da composição da blenda no comportamento reológico das blendas. Neste trabalho, o efeito da composição da blenda PP/HDPE e da temperatura no comportamento viscoelástico linear destas blendas foi estudado. De acordo com os resultados obtidos, o efeito da adição de três agentes compatibilizantes na morfologia da blenda PP/HDPE e nos valores de tensão interfacial entre PP e HDPE foram analisados.

Polímeros: Ciência e Tecnologia, vol. 11, no 4, p. 201-212, 2001 


\section{Experimental}

\section{Materiais}

Os polímeros utilizados neste trabalho foram o polipropileno (PP) e o polietileno de alta densidade (HDPE), fornecidos pela Polibrasil e pela Ipiranga Petroquímica, respectivamente. Os agentes compatibilizantes utilizados foram o terpolímero etilenopropileno-dieno (EPDM), fornecido pela Du Pont, o copolímero tribloco estireno-etileno/butileno-estireno (SEBS) fornecido pela Shell e o copolímero etilenoacetato de vinila (EVA) fornecido pela Scientific Polymer. As propriedades dos polímeros e dos agentes compatibilizantes estão apresentadas nas Tabelas 1 e 2 , respectivamente.

\section{Obtenção das Blendas}

As blendas de PP/HDPE não compatibilizadas foram obtidas variando-se a sua concentração em massa (PP/HDPE-99/1, 95/5, 90/10, 85/25, 80/20, 75/ 25 e 70/30). As blendas de PP/HDPE compatibilizadas foram preparadas mantendo-se a concentração em massa constante (PP/HDPE-80/20). A concentração de agente compatibilizante (EPDM, SEBS e EVA) variou de 2 a $20 \%$ em massa, em relação à fase dispersa.

Todas as blendas foram obtidas numa extrusora de rosca dupla Werner Pfeiderer, modelo ZSK-30 com seis zonas de temperaturas que variaram de 170 a $210^{\circ} \mathrm{C}$ ao longo do barril da extrusora. As blendas

Tabela 1. Massa molar, polidispersidade e viscosidades de cisalhamento zero a 180,200 e $220^{\circ} \mathrm{C}$ dos polímeros utilizados.

\begin{tabular}{cccccc}
\hline Polímeros & $\begin{array}{c}\overline{\boldsymbol{M}}_{w} \\
(\mathrm{~g} / \mathbf{m o l})\end{array}$ & $\overline{\boldsymbol{M}}_{w} / \overline{\boldsymbol{M}}_{\boldsymbol{n}}$ & $\begin{array}{c}\left(\boldsymbol{\eta}_{\mathbf{0}}\right) \mathbf{a} \\
\mathbf{1 8 0}^{\circ} \mathbf{C} \\
(\mathbf{P a . s})\end{array}$ & $\begin{array}{c}\left(\boldsymbol{\eta}_{\mathbf{0}}\right) \mathbf{a} \\
\mathbf{2 0 0}^{\circ} \mathbf{C} \\
(\mathbf{P a . s})\end{array}$ & $\begin{array}{c}\left(\boldsymbol{\eta}_{\mathbf{0}}\right) \mathbf{a} \\
\mathbf{2 2 0}^{\circ} \mathbf{C} \\
(\mathbf{P a} . \mathbf{s})\end{array}$ \\
\hline PP & 340.000 & 4,5 & 27.000 & 17.200 & 10.700 \\
HDPE & 140.000 & 3,2 & 10.900 & 9.040 & 8.050 \\
\hline
\end{tabular}

Tabela 2. Massa molar e polidispersidade dos agentes compatibilizantes utilizados.

\begin{tabular}{ccc}
\hline Compatibilizantes & $\begin{array}{c}\overline{\boldsymbol{M}}_{w} \\
(\mathbf{g} / \mathbf{m o l})\end{array}$ & $\overline{\boldsymbol{M}}_{w} / \overline{\boldsymbol{M}}_{n}$ \\
\hline EPDM & 320.000 & 1,88 \\
EVA & 46.000 & 1,92 \\
SEBS & 74.000 & 1,03 \\
\hline
\end{tabular}

compatibilizadas foram obtidas em duas etapas. Primeiramente, o agente compatibilizante foi misturado ao HDPE e, em seguida, este produto foi adicionado ao PP.

\section{Medidas reológicas}

As amostras utilizadas nas medidas reológicas consistem de discos com diâmetro de $25 \mathrm{~mm}$ e espessura de $1,0 \mathrm{~mm}$. Estas amostras foram obtidas por prensagem a quente dos materiais em uma placa de aço inox agindo como molde vazado, utilizando-se uma prensa hidráulica, a uma temperatura de aproximadamente $200^{\circ} \mathrm{C}$, sob uma pressão de fechamento de aproximadamente $100 \mathrm{Kgf} / \mathrm{cm}^{2}$, por um período de 30 minutos.

Os ensaios de cisalhamento oscilatório de tensão controlada foram realizados utilizando-se um reômetro da marca Rheometric Scientific SR-5000. A configuração de placas paralelas foi utilizada com um gap de aproximadamente $0,6 \mathrm{~mm}$. Os ensaios foram realizados com as blendas não compatibilizadas nas temperaturas de 180,200 e $220^{\circ} \mathrm{C}$ e para as blendas compatibilizadas na temperatura de $220^{\circ} \mathrm{C}$, em atmosfera de nitrogênio. Todos os ensaios foram realizados na região de viscoelasticidade linear dos polímeros, variando-se a freqüência de 0,01 a $100 \mathrm{rad} / \mathrm{s}$.

As viscosidades de cisalhamento zero das fases necessárias para o cálculo da tensão interfacial foram determinadas através de análises de Carreau ${ }^{[15]}$.

\section{Análises Morfológicas}

A morfologia de todas as blendas foi caracterizada por análises de Microscopia Eletrônica de Varredura (MEV). O equipamento utilizado foi um microscópio eletrônico da marca "Cambridge", modelo "Stereoscan 240". Amostras com as mesmas características das amostras utilizadas nas medidas reológicas foram fraturadas em nitrogênio líquido, para análise das seções transversais, e recobertas com ouro utilizando-se um "sputter coater" da marca Balzers.

O raio da fase dispersa, assim como sua fração volumétrica foram determinados pela análise das micrografias. Aproximadamente 300 partículas foram consideradas no cálculo destes parâmetros. Para o cálculo do tamanho médio da fase dispersa, foi utilizada a correção de Saltycov ${ }^{[16]}$ que leva em consideração a polidispersividade das amostras e o fato de 
que a fratura na amostra nem sempre ocorre no máximo diâmetro das partículas.

\section{Resultados e Discussão}

\section{Blendas não compatibilizadas}

Efeito da composição na morfologia das blendas PP/HDPE.

A Figura 1 apresenta a morfologia da blenda PP/ HDPE (80/20). Pode-se observar uma morfologia de dispersão de gotas de HDPE em uma matriz contínua de PP. Todas as amostras utilizadas em análises reológicas, compatibilizadas ou não, apresentaram este mesmo tipo de morfologia.

A Figura 2 e a tabela 3 apresentam raio numérico médio $\left(R_{n}\right)$ e o raio volumétrico médio $\left(R_{v}\right)$ da fase dispersa em função da concentração de HDPE na blenda PP/HDPE. O raio numérico médio $\left(\mathrm{R}_{\mathrm{n}}\right)$ foi calculado utilizando-se a equação (7) e o raio volumétrico médio $\left(\mathrm{R}_{\mathrm{v}}\right)$ foi calculado utilizando-se a seguinte equação:

$$
R_{v}=\frac{\sum_{i}\left(R_{i} \phi_{i}\right)}{\sum_{i} \phi_{i}}
$$

sendo $R_{i} o$ raio da i-ésima gota e $\phi_{i}$ a fração volumétrica da i-ésima gota.

Na Figura 2, os valores obtidos estão representados pelos símbolos e a linha contínua é apenas um guia para os olhos. Pode-se observar que tanto o raio volumétrico médio $\left(\mathrm{R}_{\mathrm{v}}\right)$ quanto o raio numérico médio $\left(R_{n}\right)$ da fase dispersa aumentam com o aumento da concentração de HDPE na blenda PP/HDPE. Este

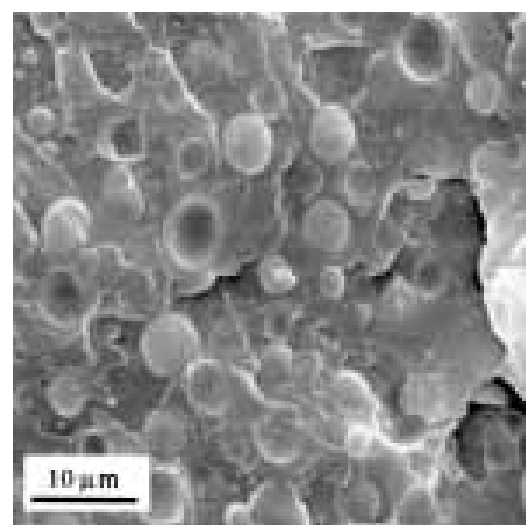

Figura 1. Morfologia da blenda PP/HDPE (80/20)

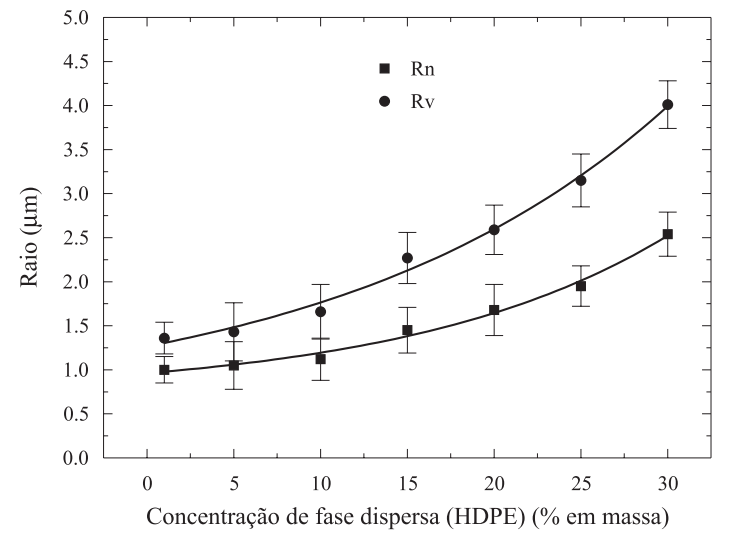

Figura 2. Raio volumétrico médio $\left(R_{v}\right)$ e raio numérico médio $\left(R_{n}\right)$ da fase dispersa em função da concentração de HDPE na blenda PP/HDPE.

Tabela 3. Raio numérico médio $\left(\mathrm{R}_{\mathrm{n}}\right)$ e o raio volumérico médio $\left(\mathrm{R}_{\mathrm{v}}\right)$ da fase dispersa e razão $R_{v} / R_{n}$.

\begin{tabular}{cccc}
\hline PP/HDPE & $\mathbf{R}_{\mathrm{n}}(\mu \mathbf{m})$ & $\mathbf{R}_{\mathrm{v}}(\boldsymbol{\mu m})$ & $\mathbf{R}_{\mathrm{v}} / \mathbf{R}_{\mathrm{n}}$ \\
\hline $99 / 1$ & $1,00 \pm 0,15$ & $1,36 \pm 0,18$ & 1,36 \\
$95 / 5$ & $1,05 \pm 0,27$ & $1,43 \pm 0,33$ & 1,36 \\
$90 / 10$ & $1,12 \pm 0,24$ & $1,66 \pm 0,31$ & 1,48 \\
$85 / 15$ & $1,45 \pm 0,26$ & $2,27 \pm 0,29$ & 1,56 \\
$80 / 20$ & $1,68 \pm 0,29$ & $2,59 \pm 0,28$ & 1,54 \\
$75 / 25$ & $1,95 \pm 0,23$ & $3,15 \pm 0,30$ & 1,61 \\
$70 / 30$ & $2,54 \pm 0,25$ & $4,01 \pm 0,27$ & 1,59 \\
\hline
\end{tabular}

fato pode estar relacionado com o aumento da coalescência devido ao aumento da concentração da fase dispersa. A coalescência é diretamente proporcional ao quadrado da concentração da fase dispersa ${ }^{[17]}$. À medida que a concentração de partículas aumenta, decresce a distância entre as partículas, facilitando o contato entre estas, resultando em coalescência.

Analisando-se a Figura 2, pode-se observar que o aumento do raio das partículas apresenta-se mais significativo somente a partir da concentração de $10 \%$ de HDPE adicionado à blenda PP/HDPE. Wallheinke et al. ${ }^{[18]}$ observaram em seus estudos com blendas de poliuretano termoplástico/polipropileno (TPU/PP) e poliuretano termoplástico/polietileno (TPU/PE) (para concentrações variando de 0,1 a $20 \%$ em massa de fase dispersa) que um significativo aumento da coalescência é observado para uma concentração muito mais baixa de fase dispersa (aproximadamente $1 \%$ ). O processo de coalescência pode explicar as

Polímeros: Ciência e Tecnologia, vol. 11, no 4, p. 201-212, 2001 
diferenças entre os resultados de Wallheinke et al. ${ }^{[18]}$ e os resultados obtidos neste trabalho. A coalescência depende de dois parâmetros principais: do número de colisões das gotas e da habilidade da blenda de drenar o filme da fase matriz existente entre duas gotas da fase dispersa até que uma espessura crítica é alcançada para que a coalescência ocorra ${ }^{[19,20]}$ : quando o número de colisões das gotas da fase dispersa e a habilidade de drenagem do filme é alta, a coalescência pode ocorrer em baixas concentrações de fase dispersa. O número de colisões das gotas é proporcional ao quadrado da concentração da fase dispersa e é inversamente proporcional ao tamanho da gota. A drenagem do filme é um fenômeno complexo que depende da tensão interfacial entre os polímeros e é inversamente proporcional à viscosidade da matriz. Quanto menor o valor da tensão interfacial e da razão de viscosidade (fase dispersa/ matriz), menor é a habilidade de drenar o filme da fase matriz existente entre duas gotas. No caso das blendas PP/HDPE, a tensão interfacial entre PP e HDPE é muito menor que a tensão interfacial entre TPU/PP e TPU/PE (aproximadamente $1 \mathrm{mN} / \mathrm{m}$ comparado a 8,2 e 9,4 $\mathrm{mN} / \mathrm{m}$ ). Também, a razão de viscosidade de HDPE/PP estudada neste trabalho é menor comparado à razão de viscosidade para TPU/ PP e TPU/PE estudados por Wallheinke et al. $\left.{ }^{[18}\right]$ (menor que 1 comparado com 3,54). Estas diferenças no comportamento reológico e nos valores de tensão interfacial entre os polímeros podem explicar as diferenças de magnitude da coalescência observada.

Efeito da composição e da temperatura no comportamento reológico das blendas PP/HDPE e cálculos da tensão interfacial.

As Figuras 3 e 4 apresentam os módulos de armazenamento $\left(G^{\prime}(\omega)\right)$ e de perda $\left(G^{\prime \prime}(\omega)\right)$ das blendas PP/HDPE com diferentes composições e das fases puras quando submetidos a um cisalhamento oscilatório na temperatura de $220^{\circ} \mathrm{C}$. Analisando as Figuras 3 e 4, pode-se observar que a curva do módulo de perda das blendas quase coincide com a curva da matriz. No entanto, em baixas frequiências, o módulo de armazenamento das blendas de PP/HDPE em todas as composições é maior que o módulo de armazenamento das fases puras. Este comportamento foi observado para todas as temperaturas analisadas. Pode ser visto também que, em baixas freqüências, o módulo de armazenamento das blendas aumenta com

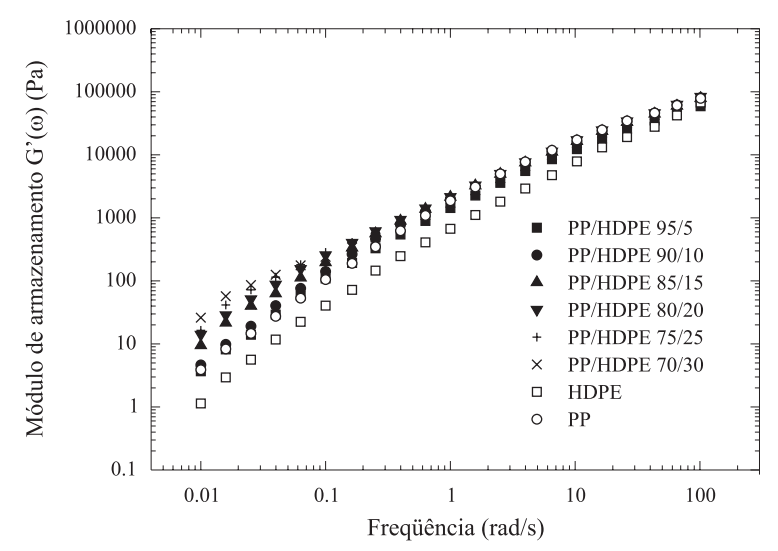

Figura 3. Módulo de armazenamento $\left(\mathrm{G}^{\prime}(\omega)\right)$ das blendas PP/HDPE com diferentes composições e das fases puras quando submetidos a um cisalhamento oscilatório na temperatura de $220^{\circ} \mathrm{C}$

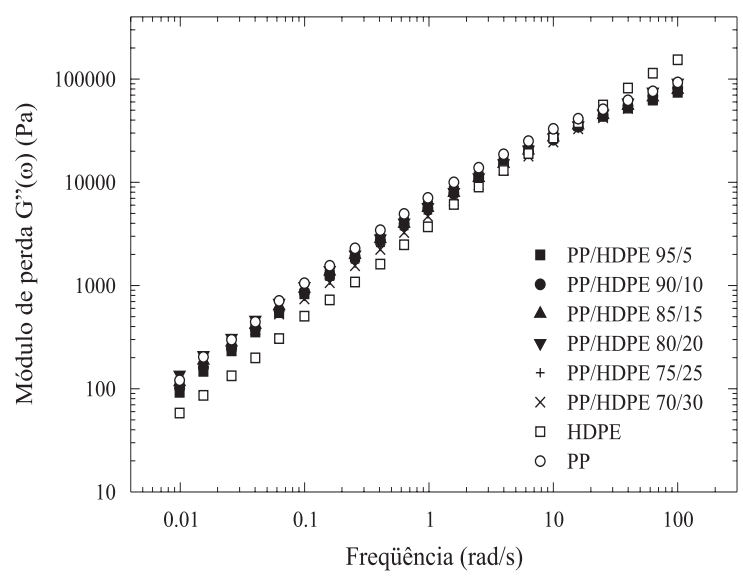

Figura 4. Módulo de perda $(G$ ' $(\omega))$ das blendas PP/HDPE com diferentes composições e das fases puras quando submetidos a um cisalhamento oscilatório na temperatura de $220^{\circ} \mathrm{C}$

o aumento da concentração da fase dispersa (HDPE). Este comportamento pode ser atribuído a um processo de relaxação das gotas da fase dispersa quando estas são levemente deformadas ${ }^{[8-13]}$. Quando a concentração da fase dispersa aumenta, é esperado que o diâmetro das gotas da fase dispersa aumente e o processo de relaxação torna-se mais lento, resultando num aumento do módulo de armazenamento da blenda.

Os espectros de relaxação ponderado das blendas PP/HDPE foram obtidos para todas as composições e temperaturas analisadas. A Figura 5 apresenta os espectros de relaxação ponderado das blendas PP/HDPE nas composições de 95/5, 85/15 e 75/25 na temperatura de $220^{\circ} \mathrm{C}$. Somente alguns espectros de relaxação ponderado das blendas PP/HDPE foram mostrados para facilitar a visualização. $\mathrm{O}$ espectro de relaxação pode ser calculado através de vários métodos $^{[21-23]}$. Neste trabalho, todos os espectros de relaxação foram obtidos através de dados experimen- 


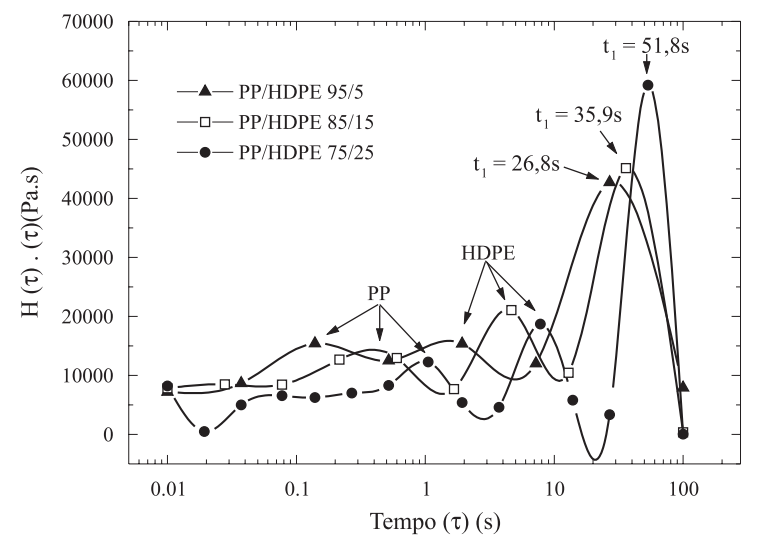

Figura 5. Espectros de relaxação ponderado das blendas PP/HDPE nas concentrações de $95 / 5,85 / 15$ e $75 / 25$ a $220^{\circ} \mathrm{C}$

tais do módulo dinâmico de armazenamento, utilizando-se um método de regressão não linear, disponível no programa de análise do reômetro SR-5000 da Rheometrics. Observa-se na Figura 5 que os espectros de relaxação ponderado das blendas apresentam 3 picos característicos. Dois deles estão associados à relaxação das fases puras e o terceiro está relacionado à relaxação da forma das gotas da fase dispersa. Este tipo de espectro de relaxação foi observado para todas as blendas aqui estudadas e em todas as temperaturas.

As Tabelas 4, 5 e 6 apresentam o tempo de relaxação associado à relaxação da forma das gotas da fase dispersa da blenda PP/HDPE obtido experimentalmente para todas as blendas estudadas nas temperaturas de 180,200 e $220^{\circ} \mathrm{C}$, respectivamente. Analisando-se as Tabelas 4, 5 e 6, pode ser visto que o tempo de relaxação associado à relaxação da forma das gotas da fase dispersa não foi possível de ser observado na blenda PP/HDPE (99/1) para todas as temperaturas. Este comportamento já foi observado por outros pesquisadores ${ }^{[12]}$ e está relacionado à baixa concentração da fase dispersa que apresenta um tempo relaxação muito pequeno, tornando a distinção entre este pico e os picos associados à relaxação das fases extremamente difícil.

Analisando-se as Tabelas 4, 5 e 6 também pode ser visto que o tempo de relaxação associado à relaxação da forma das gotas da fase dispersa aumenta com o aumento da concentração de HDPE na blenda. Este fato pode ser explicado ao lembrar-se de que o tempo de relaxação da forma das gotas da fase dispersa é devido a um balanço entre duas forças ${ }^{[10,24]}$ : uma força cisalhante, proporcional a $\eta \dot{\gamma}$ (onde $\eta$ é a viscosidade e $\dot{\gamma}$ é a taxa de cisalhamento) que tende a deformar as partículas da fase dispersa e uma força coesiva, proporcional à razão $\gamma / d$ (onde $\gamma$ é a tensão interfacial entre dois polímeros e $d$ é o diâmetro da partícula) que contribui para a retração da fase dispersa para a forma esférica. Quando a concentração da fase dispersa aumenta, é esperado que o diâmetro da fase dispersa aumente. Portanto, a força proporcional à tensão interfacial decresce e o tempo de relaxação da forma da fase dispersa aumenta.

Os valores do tempo de relaxação da forma das gotas da fase dispersa para cada blenda obtidos experimentalmente nas temperaturas de 180,200 e $220^{\circ} \mathrm{C}$ foram utilizados no cálculo da tensão interfacial entre PP e HDPE usando as análises de Gramespacher e Meissner ${ }^{[1]}$. Os valores de tensão interfacial calculados também estão apresentados nas Tabelas 4, 5 e 6. Pode-se observar que os valores de tensão interfacial entre PP e HDPE variaram de 1,53 a 3,68 $\mathrm{mN} / \mathrm{m}\left(180^{\circ} \mathrm{C}\right), 1,10$ a $2,81 \mathrm{mN} / \mathrm{m}\left(200^{\circ} \mathrm{C}\right)$ e 1,01 a $2,32 \mathrm{mN} / \mathrm{m}\left(180^{\circ} \mathrm{C}\right)$. Observa-se também que para as composições variando de $85 / 15$ a $75 / 25$, a tensão interfacial parece apresentar um valor praticamente constante. Estes resultados indicam que existe uma faixa de composições onde é possível utilizar-se as análises de Gramespacher e Meissner ${ }^{[1]}$ para a obtenção da tensão interfacial entre polímeros usando medidas reológicas. Se a concentração é baixa (no caso deste estudo $\leq 10 \%$ ), o tempo de relaxação da forma das gotas da fase dispersa é pequeno e a distinção entre este pico e os picos associados à relaxação das fases torna-se difícil. Efeitos de sobreposição de picos pode tornar a obtenção do tempo de relaxação da forma das gotas da fase dispersa pouco confiável ${ }^{[12]}$. Por outro lado, se a concentração da fase dispersa é

Tabela 4. Tempos de relaxação da forma das gotas da fase dispersa $\left(\tau_{1}\right)$ e tensão interfacial $(\gamma)$ entre PP e HDPE a $180^{\circ} \mathrm{C}$

\begin{tabular}{ccc}
\hline PP/HDPE & $\tau_{\mathbf{1}}(\mathbf{s})$ & $\boldsymbol{\gamma}(\mathbf{m N} / \mathbf{m})$ \\
\hline $99 / 1$ & não observado & - \\
$95 / 5$ & 37,83 & $1,53 \pm 0,40$ \\
$90 / 10$ & 39,81 & $1,89 \pm 0,41$ \\
$85 / 15$ & 39,81 & $2,69 \pm 0,52$ \\
$80 / 20$ & 59,67 & $2,57 \pm 0,44$ \\
$75 / 25$ & 76,32 & $2,64 \pm 0,32$ \\
$70 / 30$ & 79,59 & $3,68 \pm 0,37$ \\
\hline
\end{tabular}


Tabela 5. Tempos de relaxação da forma das gotas da fase dispersa $\left(\tau_{1}\right)$ e tensão interfacial $(\gamma)$ entre PP e HDPE a $200^{\circ} \mathrm{C}$

\begin{tabular}{ccc}
\hline PP/HDPE & $\boldsymbol{\tau}_{\mathbf{1}}(\mathbf{s})$ & $\boldsymbol{\gamma}(\mathbf{m N} / \mathbf{m})$ \\
\hline $99 / 1$ & não observado & - \\
$95 / 5$ & 35,94 & $1,10 \pm 0,28$ \\
$90 / 10$ & 35,94 & $1,41 \pm 0,31$ \\
$85 / 15$ & 36,31 & $1,98 \pm 0,38$ \\
$80 / 20$ & 43,29 & $2,36 \pm 0,41$ \\
$75 / 25$ & 61,62 & $2,18 \pm 0,27$ \\
$70 / 30$ & 69,31 & $2,81 \pm 0,29$ \\
\hline
\end{tabular}

Tabela 6. Tempos de relaxação da forma das gotas da fase dispersa $\left(\tau_{1}\right)$ e tensão interfacial $(\gamma)$ entre PP e HDPE a $220^{\circ} \mathrm{C}$

\begin{tabular}{ccc}
\hline PP/HDPE & $\boldsymbol{\tau}_{\mathbf{1}}(\mathbf{s})$ & $\boldsymbol{\gamma}(\mathbf{m N} / \mathbf{m})$ \\
\hline $99 / 1$ & não observado & - \\
$95 / 5$ & 26,83 & $1,01 \pm 0,26$ \\
$90 / 10$ & 31,62 & $1,09 \pm 0,24$ \\
$85 / 15$ & 35,94 & $1,44 \pm 0,27$ \\
$80 / 20$ & 39,81 & $1,72 \pm 0,31$ \\
$75 / 25$ & 51,80 & $1,72 \pm 0,22$ \\
$70 / 30$ & 55,62 & $2,32 \pm 0,26$ \\
\hline
\end{tabular}

grande (no caso deste estudo $\geq 30 \%$ ), a distância entre as partículas torna-se pequena e fenômenos de interação partícula-partícula podem afetar o comportamento reológico da blenda ${ }^{[11,12]}$.

Com o objetivo de verificar se os tempos de relaxação obtidos experimentalmente através de espectros de relaxação podem realmente ser relacionados com a relaxação da forma das gotas da fase dispersa, comparou-se estes valores com os previstos pelo modelo de emulsão de Palierne ${ }^{[7]}$ para a temperatura de $220^{\circ} \mathrm{C}$, utilizando-se a seguinte equação:

$$
\tau_{D}=\frac{R_{v} \eta_{m}}{4 \gamma} \frac{(19 K+16)(2 K+3-2 \phi(K-1))}{10(K+1)-2 \phi(5 K+2)}
$$

onde $R_{\mathrm{v}}$ é o raio volumétrico médio; $\eta_{\mathrm{m}}$ é a viscosidade da fase matriz; $\gamma$ é a tensão interfacial; $\phi$ é a fração volumétrica da fase dispersa e $K=. \eta_{\mathrm{d}} / \eta_{\mathrm{m}}$ onde $\eta_{d}$ é a viscosidade de cisalhamento zero da fase dispersa e $\eta_{\mathrm{m}}$ é a viscosidade de cisalhamento zero da matriz
As viscosidades de cisalhamento zero da matriz (PP) e da fase dispersa (HDPE) usados nos cálculos estão apresentadas na Tabela 1 e os valores de $R_{v}$ estão apresentados na Tabela 3. O valor de tensão interfacial utilizado foi calculado através de dados obtidos por Rao et al. ${ }^{[28]}$ que mediu a tensão interfacial entre polipropileno (PP) e polietileno de baixa densidade linear (LLDPE) utilizando o método da instabilidade de fibras inseridas (breaking thread). Os valores obtidos pelos pesquisadores variaram de $0,2 \mathrm{a} 2,6 \mathrm{mN} /$ $\mathrm{m}$ a $220^{\circ} \mathrm{C}$. Portanto, com o objetivo de obter um valor de tensão interfacial entre $\mathrm{PP}$ e $\mathrm{PE}$ a $220^{\circ} \mathrm{C}$ para avaliar o tempo de relaxação previsto pelo modelo de emulsão de Paliene, foi utilizada uma média de valores obtidos por Rao et al. ${ }^{[28]}$. O valor obtido foi de $\gamma=1,58 \mathrm{mN} / \mathrm{m}$.

Os valores do tempo de relaxação previsto pelo modelo de emulsão de Palierne estão apresentados na Tabela 7. Pode-se observar que os valores do tempo de relaxação previsto pelo modelo de emulsão de Palierne corroboraram com os valores experimentais somente para concentrações das blendas variando de 15 a $25 \%$ em massa de fase dispersa (HDPE). Estes resultados corroboraram com as observações feitas para os valores de tensão interfacial obtidos e confirmam a possibilidade de existência de uma faixa de composições da blenda PP/HDPE para a qual é possível a medida da tensão interfacial através de medidas reológicas. Os valores da tensão interfacial entre PP e HDPE obtidos nesta faixa de composições foi utilizada para avaliar a influência da temperatura na tensão interfacial entre PP e HDPE.

A Figura 6 apresenta a tensão interfacial entre PP e HDPE em função da temperatura. Os valores da tensão interfacial foram obtidos através da média de

Tabela 7. Valores do tempo de relaxação da forma da fase dispersa obtidos experimentalmente $\left(\tau_{1 \text { exp }}\right.$ ) e previsto pelo modelo de emulsão de Palierne $\left(\tau_{1 \text { Palierne }}\right)$

\begin{tabular}{ccc}
\hline PP/HDPE & $\tau_{1 \text { exp }}(\mathbf{s})$ & $\tau_{1 \text { Palierne }}(\mathbf{s})$ \\
\hline $95 / 5$ & 26,83 & 19,60 \\
$90 / 10$ & 31,62 & 23,69 \\
$85 / 15$ & 35,94 & 33,75 \\
$80 / 20$ & 39,81 & 40,18 \\
$75 / 25$ & 51,80 & 51,07 \\
$70 / 30$ & 55,62 & 68,04 \\
\hline
\end{tabular}




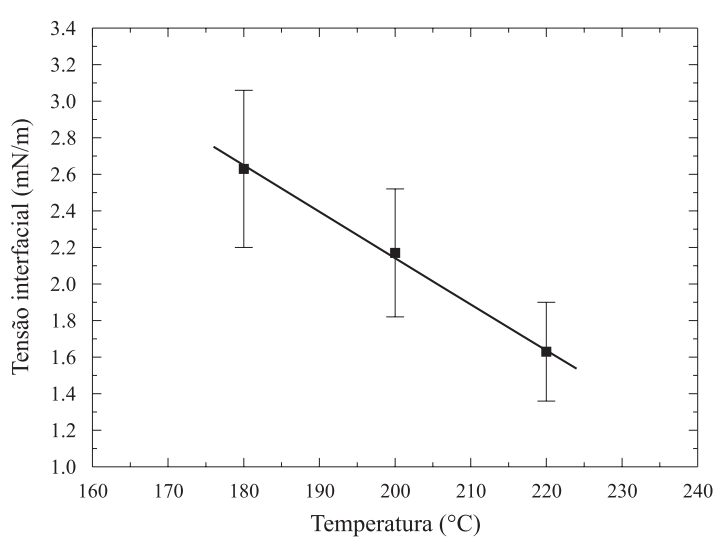

Figura 6. Tensão interfacial entre PP e HDPE em função da temperatura

valores de tensão interfacial calculada através das análises reológicas das blendas nas concentrações de 15,20 e $25 \%$ em massa de fase dispersa. Os símbolos representam os dados experimentais e a linha representa a melhor correlação obtida por regressão linear. Pode ser visto que a tensão interfacial decresce linearmente com a temperatura seguindo a equação abaixo:

$$
\gamma=7,20-0,025 T
$$

onde $\gamma$ é a tensão interfacial e $T$ é a temperatura.

O decréscimo linear da tensão interfacial com a temperatura é esperado termodinamicamente e tem sido reportado por muitos pesquisadores para diferentes pares de polímeros ${ }^{[25,26]}$. O coeficiente de temperatura $(\partial \gamma / \partial T)$ obtido foi de $\left(-0,025 \times 10^{-3} \mathrm{~N} / \mathrm{mK}\right) \mathrm{e}$ corroborou com valores obtidos para outros pares de polímeros $^{[27]}$.

A seguir, o estudo do efeito da adição de agente compatibilizante na morfologia da blenda PP/HDPE e no valor da tensão interfacial entre PP e HDPE será apresentado. Neste estudo, utilizou-se a blenda PP/ HDPE 80/20 devido ao fato desta apresentar uma composição que se apresenta dentro da faixa composições no qual é possível a medida da tensão interfacial através de medidas reológicas.

\section{Blendas compatibilizadas}

Efeito da adição de agente compatibilizante na morfologia das blendas PP/HDPE

A Figura 7 apresenta o raio numérico médio da fase dispersa em função da concentração de EPDM, EVA e SEBS adicionada à blenda PP/HDPE 80/20. Os valores obtidos estão representados pelos símbolos e a linha contínua é apenas um guia para os olhos.

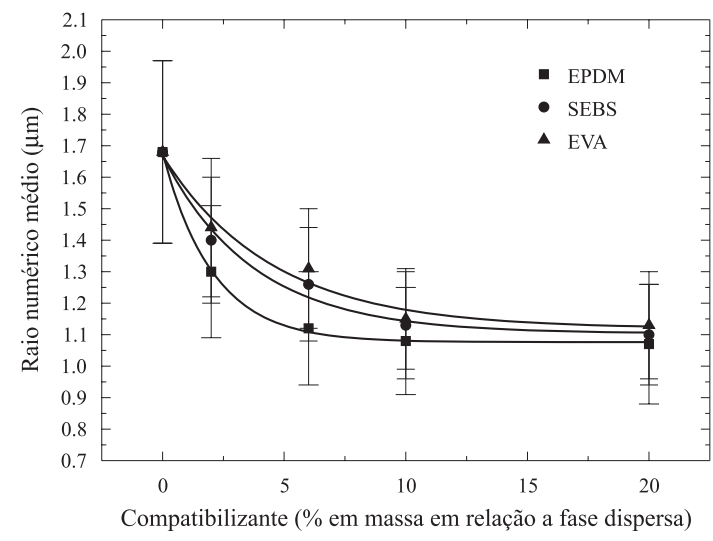

Figura 7. Raio numérico médio da fase dispersa em função da concentração de EPDM, EVA e SEBS adicionada a blenda PP/HDPE 80/20

Pode-se notar que o raio da fase dispersa decresce em função da concentração de agente compatibilizante adicionado até uma certa concentração onde, a partir desta, o raio apresenta-se praticamente constante. Esta concentração característica é conhecida como concentração crítica ou concentração de saturação, pois, segundo Favis ${ }^{[6]}$, a partir desta concentração a interface apresenta-se saturada pelo copolímero.

A seguinte equação exponencial apresenta uma boa estimativa da dependência da média do raio da fase dispersa em função da concentração de copolímero adicionado $^{[10]}$ :

$$
\frac{R_{n c}-R_{\infty}}{R_{0}-R_{\infty}}=\exp \left(-n_{1} c\right)
$$

onde $\mathrm{R}_{\mathrm{nc}}$ é a média do raio da fase dispersa a uma concentração de agente compatibilizante $c, R_{o}$ é a média do raio da fase dispersa para a blenda sem agente compatibilizante, $R_{\infty}$ é uma constante que corresponde ao raio para uma concentração infinita de agente compatibilizante, e $\mathrm{n}_{1}$ é uma constante que determina a eficiência do compatibilizante como agente emulsificante.

Com o objetivo de comparar a eficiência dos três compatibilizantes, foi considerada a concentração $\mathrm{c}_{\mathrm{R} 0,05}$ que corresponde a uma concentração de agente compatibilizante para o qual a relação $\left(\mathrm{R}_{\mathrm{nc}}-\mathrm{R}_{\infty}\right)$ / $\left(R_{o}-R_{\infty}\right.$ ) (variação do valor do $R_{n c}$ em relação ao raio correspondente a uma concentração infinita de agente compatibilizante $\mathrm{R}_{\infty}$ ) seja igual a 0,05 ou $5 \%$. Para concentrações maiores que $c_{\mathrm{R} 0,05}$, o decréscimo do valor de $\mathrm{R}_{\mathrm{nc}}$ pode ser considerado negligenciável. A Tabela 8 apresenta os valores de $\mathrm{R}_{\infty}$, e $\mathrm{n}_{1}$ da equação 
(11), bem como a concentração $c_{\mathrm{R} 0,05}$ para a blenda PP/HDPE 80/20 compatibilizada com os 3 agentes compatibilizantes. Pode-se observar que o valor da constante que determina a eficiência do compatibilizante como agente emulsificante $\left(\mathrm{n}_{1}\right)$ é maior para o EPDM que para o SEBS e EVA. O valor do raio para uma concentração infinita $\left(\mathrm{R}_{\infty}\right)$, bem como a concentração $\mathrm{c}_{\mathrm{R} 0,05}$ apresentou-se menor para o EPDM. Estes resultados indicam uma maior eficiência do EPDM em relação ao SEBS e EVA como agente emulsificante para a blenda PP/HDPE. Em outras palavras, é necessária uma menor concentração de EPDM comparada à concentração de SEBS e EVA para que o tamanho da fase dispersa alcance o menor valor.

A maior eficiência do EPDM como emulsificante para as blendas PP/HDPE em comparação ao SEBS e EVA pode estar relacionada com os diferentes componentes formadores dos copolímeros estudados. O EPDM possui em sua cadeia polimérica, o bloco (PE) e o bloco (PP) que apresentam boa interação com os componentes das blendas PP/HDPE. O EVA e o SEBS possuem em suas cadeias poliméricas, o bloco (PE) que apresenta boa interação com o componente HDPE da blenda PP/PEAD. No entanto, o bloco (VA) do EVA e os blocos (S) do SEBS não apresentam boa interação com o PP das blendas PP/HDPE, podendo influenciar na menor eficiência destes dois copolímeros como emulsificantes das blendas PP/HDPE ${ }^{[29]}$. $\mathrm{O}$ fato do bloco (VA) do EVA ser polar pode contribuir para o aumento da tensão interfacial entre PP e HDPE, resultando numa menor eficiência do EVA como emulsificante para a blenda PP/HDPE, em comparação ao SEBS que apresenta o bloco (S) apolar como o PP.

Uma outra possibilidade para a maior eficiência do EPDM como emulsificante para as blendas PP/ HDPE seria a sua maior massa molar. No entanto, Matos et al. ${ }^{[30]}$ em seu estudo com blendas de poliestireno (PS)/copolímero de etileno-propileno (EPR) compatibilizadas com SEBS, mostraram que a massa molar do SEBS não afetou a concentração

\begin{tabular}{cccc} 
Tabela 8. Valores de $\mathrm{R}_{\infty}, \mathrm{n}_{1} \mathrm{e} \mathrm{de}_{\mathrm{R} 0,05}$ para os 3 agentes compatibilizantes \\
\hline Compatibilizante & $\mathbf{R}_{\infty}(\boldsymbol{\mu m})$ & $\mathbf{n}_{\mathbf{1}}$ & $\mathbf{C}_{\mathbf{R} 0.05}(\%)$ \\
\hline EPDM & 1,11 & 0,51 & 5,9 \\
EVA & 1,18 & 0,30 & 9,9 \\
SEBS & 1,16 & 0,34 & 8,7 \\
\hline
\end{tabular}

crítica de agente compatibilizante ou o tamanho da fase dispersa no equilíbrio.

Efeito da adição de agente compatibilizante no valor da tensão interfacial entre PP e HDPE.

A Figura 8 apresenta os espectros de relaxação da blenda PP/HDPE (80/20) com adição de 6, 10 e $20 \%$ de EPDM. Todas as blendas PP/HDPE (80/20) compatibilizadas com EPDM, SEBS e EVA apresentaram este mesmo comportamento. Pode-se observar que os espectros de relaxação apresentam três picos característicos: dois deles relativos aos tempos de relaxação das fases. O terceiro pico apresenta valores da mesma ordem de magnitude do tempo de relaxação da forma das gotas da fase dispersa obtidas para as blendas não-modificadas. Portanto, este terceiro pico está associado ao tempo de relaxação da forma das gotas da fase dispersa.

Neste trabalho, devido à baixa concentração de agente compatibilizante (máximo de $4 \%$ em massa em relação à blenda como um todo) usado para modificar a blenda PP/HDPE (80/20), assumiu-se que o modelo descrito por Gramespacher e Meissner ${ }^{[1]}$ poderia ser utilizado para avaliar a tensão interfacial entre PP e HDPE, utilizando-se blendas de PP/HDPE compatibilizadas. A contribuição da fase dispersa foi considerada como a soma da contribuição da fase dispersa (HDPE) e a contribuição do agente compatibilizante (EPDM, EVA e SEBS). Este tipo de consideração já foi assumido por outros pesquisadores ${ }^{[9,10]}$.

As Tabelas 9, 10 e 11 apresentam os tempos de relaxação da forma das gotas da fase dispersa obtidos através dos espectros de relaxação das blendas compatibilizadas com EPDM, EVA e SEBS, respectivamente.

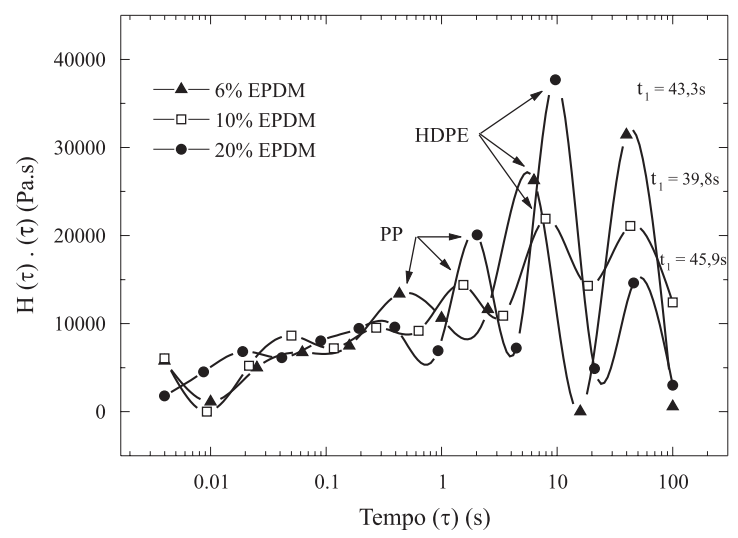

Figura 8. Espectros de relaxação da blenda PP/HDPE (80/20) com adição de 6, 10 e $20 \%$ de EPDM 
Tabela 9. Tempos de relaxação da forma das gotas da fase dispersa das blendas compatibilizadas com EPDM e tensão interfacial entre PP e HDPE a $220^{\circ} \mathrm{C}$

\begin{tabular}{ccc}
\hline $\mathbf{E P D M}(\boldsymbol{\%})$ & $\boldsymbol{\tau}_{\mathbf{1}}(\mathbf{s})$ & $\boldsymbol{\gamma}(\mathbf{m N} / \mathbf{m})$ \\
\hline 0 & 39,81 & $1,72 \pm 0,31$ \\
2 & 39,81 & $1,38 \pm 0,23$ \\
6 & 39,81 & $1,21 \pm 0,20$ \\
10 & 43,29 & $1,18 \pm 0,20$ \\
20 & 45,87 & $1,16 \pm 0,22$ \\
\hline
\end{tabular}

Tabela 10. Tempos de relaxação da forma das gotas da fase dispersa das blendas compatibilizadas com EVA e tensão interfacial entre PP e HDPE a $220^{\circ} \mathrm{C}$

\begin{tabular}{ccc}
\hline $\mathbf{E V A}(\boldsymbol{\%})$ & $\boldsymbol{\tau}_{\mathbf{1}}(\mathbf{s})$ & $\boldsymbol{\gamma}(\mathbf{m N} / \mathbf{m})$ \\
\hline 0 & 39,81 & $1,72 \pm 0,31$ \\
2 & 39,81 & $1,50 \pm 0,24$ \\
6 & 39,81 & $1,38 \pm 0,21$ \\
10 & 43,29 & $1,31 \pm 0,20$ \\
20 & 43,29 & $1,32 \pm 0,21$ \\
\hline
\end{tabular}

Tabela 11. Tempos de relaxação da forma das gotas da fase dispersa das blendas compatibilizadas com SEBS e tensão interfacial entre PP e HDPE a $220^{\circ} \mathrm{C}$

\begin{tabular}{ccc}
\hline SEBS $(\%)$ & $\tau_{\mathbf{1}}(\mathbf{s})$ & $\boldsymbol{\gamma}(\mathbf{m N} / \mathbf{m})$ \\
\hline 0 & 39,81 & $1,72 \pm 0,31$ \\
2 & 39,81 & $1,47 \pm 0,22$ \\
6 & 39,81 & $1,35 \pm 0,21$ \\
20 & 43,29 & $1,25 \pm 0,20$ \\
\hline
\end{tabular}

Pode-se observar que os tempos de relaxação da forma das gotas da fase dispersa aumentam com o aumento da concentração dos três agentes compatibilizante estudados. Este fato é esperado, pois conforme dito anteriormente, o tempo de relaxação da forma das gotas da fase dispersa é devido a um balanço entre uma força cisalhante e uma força coesiva, proporcional à razão entre a tensão interfacial e o diâmetro da partícula da fase dispersa ${ }^{[10,24]}$. Quando EPDM, SEBS ou EVA é adicionado a blenda PP/HDPE, é esperado que a tensão interfacial entre PP e HDPE decresça. A força proporcional à tensão

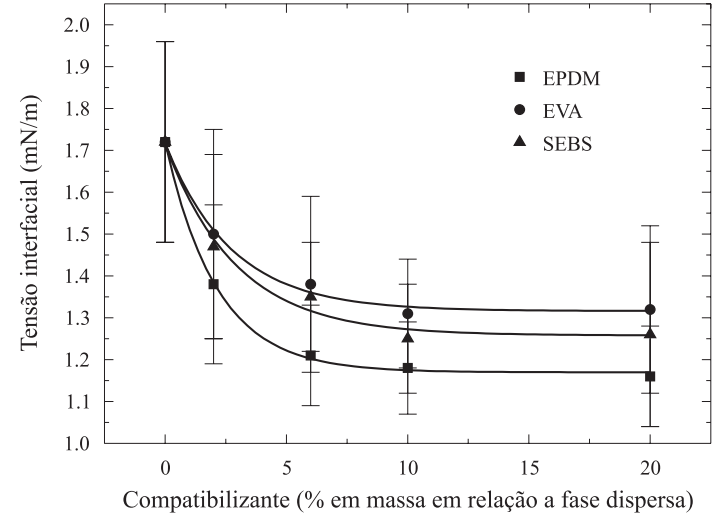

Figura 9. Tensão interfacial entre PP/HDPE em função da concentração de EPDM, EVA e SEBS a $220^{\circ} \mathrm{C}$.

interfacial decresce e o tempo de relaxação da forma da fase dispersa aumenta.

A Figura 9 apresenta os valores da tensão interfacial entre PP/HDPE em função da concentração de EPDM, EVA e SEBS a $220^{\circ} \mathrm{C}$. Pode-se notar que a tensão interfacial decresce em função da concentração de agente compatibilizante adicionado até uma certa concentração de agente compatibilizante onde, a partir desta, a tensão interfacial apresenta-se praticamente constante.

Uma equação exponencial semelhante àquela utilizada na análise do raio da fase dispersa apresenta uma boa estimativa da dependência da tensão interfacial em função da concentação de copolímero adicionado $^{[10]}$ :

$$
\frac{\gamma_{c}-\gamma_{\infty}}{\gamma_{0}-\gamma_{\infty}}=\exp \left(-n_{2} c\right)
$$

onde $\gamma_{c}$ é a tensão interfacial a uma concentração de agente compatibilizante $c$ adicionada, $\gamma_{0}$ é a tensão interfacial entre PP e HDPE sem adição de agente compatibilizante, $\gamma_{\infty}$ é uma constante que corresponde à tensão interfacial em uma concentração infinita de agente compatibilizante, e $\mathrm{n}_{2}$ é uma constante que determina a eficiência do compatibilizante no decréscimo da tensão interfacial.

Com o objetivo de comparar a eficiência dos três compatibilizantes, foi considerada a concentração $\mathrm{c}_{\gamma_{0,05}}$ no qual $\left(\gamma_{\mathrm{nc}}-\gamma_{\infty}\right) /\left(\gamma_{\mathrm{o}}-\gamma_{\infty}\right)=0,05$. Para concentrações maiores que $c_{\mathrm{R} 0,05}$, o decréscimo do valor de $\gamma_{\mathrm{nc}}$ pode ser considerado negligenciável. A Tabela 12 apresenta os valores de $\gamma_{\infty}$, e $n_{2}$ da equação (12), bem como a concentração $c_{\gamma 0,05}$ para os 3 agentes compatibilizantes. 
Tabela 12. Valores de $\gamma_{\infty}, \mathrm{n}_{2}$ e $\mathrm{c}_{\gamma 0,05}$ para os 3 agentes compatibilizantes

\begin{tabular}{cccc}
\hline Compatibilizante & $\boldsymbol{\gamma}_{\infty}(\boldsymbol{\mu m})$ & $\mathbf{n}_{\mathbf{2}}$ & $\mathbf{C}_{\boldsymbol{\gamma} 0.05}$ \\
\hline EPDM & 1,21 & 0,53 & 5,6 \\
EVA & 1,33 & 0,33 & 9,2 \\
SEBS & 1,28 & 0,34 & 8,8 \\
\hline
\end{tabular}

Pode-se observar que o valor da constante que determina a eficiência do compatibilizante no decréscimo da tensão interfacial entre PP e HDPE $\left(\mathrm{n}_{2}\right)$ é maior para o EPDM que para o SEBS e EVA. O valor da tensão interfacial entre PP e HDPE para uma concentração infinita $\left(\gamma_{\infty}\right)$, bem como a concentração $c_{\gamma 0,05}$ apresentouse menor para o EPDM comparado com SEBS e EVA. Estes resultados corroboram com os resultados morfológicos e indicam uma maior eficiência do EPDM em relação ao SEBS e EVA no decréscimo da tensão interfacial entre PP e HDPE. Em outras palavras, é necessária uma menor concentração de EPDM comparada à concentração de SEBS e EVA para que a tensão interfacial entre PP e HDPE alcance o menor valor.

\section{Conclusões}

Blendas de PP/HDPE sem adição de agente compatibilizante com concentrações variando de 1 a $30 \%$ em massa de HDPE foram estudadas. Todas as blendas apresentaram uma morfologia de dispersão de gotas de HDPE em uma matriz contínua de PP. O raio volumétrico médio $\left(\mathrm{R}_{\mathrm{v}}\right)$ e o raio numérico médio $\left(\mathrm{R}_{\mathrm{n}}\right)$ da fase dispersa aumentaram com o aumento concentração de HDPE na blenda PP/HDPE. Este fato pode estar relacionado com o aumento da coalescência devido ao aumento da concentração da fase dispersa.

A tensão interfacial entre PP e HDPE foi obtida através dos espectros de relaxação das blendas PP/HDPE com diferentes composições, seguindo as análises de Gramespacher e Meissner ${ }^{[1]}$. Os resultados indicaram que existe uma faixa de composições da blenda PP/HDPE para a qual é possível utilizar-se as análises de Gramespacher e Meissner ${ }^{[1]}$ para a obtenção da tensão interfacial entre polímeros utilizando-se medidas reológicas. Este comportamento está relacionado às diferenças no comportamento reológico das blendas com diferentes composições.

Os valores de tensão interfacial entre PP e HDPE, obtidos através da faixa de composições para a qual é possível utilizar-se as análises de Gramespacher e Meissner ${ }^{[1]}$, para a obtenção da tensão interfacial entre polímeros utilizando-se medidas reológicas, foram utilizados no estudo da influência da temperatura nos valores de tensão interfacial entre PP e HDPE. Observou-se que a tensão interfacial entre PP e HDPE decresce linearmente com a temperatura.

O efeito da adição de agente compatibilizante (EPDM, SEBS e EVA) na morfologia da blenda PP/HDPE 80/20 e nos valores de tensão interfacial entre PP e HDPE também foi estudado. O raio médio da fase dispersa da blenda decresceu em função da concentração de agente compatibilizante adicionado até uma certa concentração onde, a partir desta, o raio apresentou-se praticamente constante. Os resultados indicaram uma maior eficiência do EPDM em relação ao SEBS e EVA como agente emulsificante para a blenda PP/HDPE.

A tensão interfacial entre PP e HDPE foi obtida através dos espectros de relaxação das blendas PP/HDPE com adição de agente compatibilizante, seguindo as análises de Gramespacher e Meissner ${ }^{[1]}$. Os valores de tensão interfacial entre PP e HDPE decresceram em função da concentração de agente compatibilizante adicionado até uma certa concentração de agente compatibilizante onde, a partir desta, a tensão interfacial apresentou-se praticamente constante. Estes resultados corroboraram com os resultados morfológicos e indicaram uma maior eficiência do EPDM em relação ao SEBS e EVA no decréscimo da tensão interfacial entre PP e HDPE.

\section{Agradecimentos}

Os autores agradecem à Polibrasil S. A., Ipiranga Petroquímica S. A., Shell Chemical e Du Pont pela doação de materiais e a FAPESP pelo apoio financeiro (FAPESP 97/06071-2 e FAPESP 98/01194-1).

\section{Referências Bibliográficas}

1. Grasmespacher, H.; Meissner, J. J. - Rheology, 36(6), 1127 (1992).

2. Dumoulin, M. M.; Carreau, P. J. - J. Polym. Eng. Sci, 27(20), 1627 (1987).

3. Flaris,V.; Wenig, W.; Stachurski - Materials Forum, 16(2), 181 (1992).

4. Flaris, V.; Wasiak, A.; Wenig, W. J. - Mat. Sci., 28, 1685 (1993). 
5. Blom, H. P.; Teh, J. W.; Rudin, A. - J. Appl. Polym. Sci., 61, 959 (1996).

6. Favis, B. D. - Polymer, 35(7), 1552 (1994).

7. Graebling, D.; Benkira, A.; Gallot, Y.; Muller, R. - European Polymer Journal, 30, 301 (1994).

8. Choi, S. J.; Showalter, W. R. - Physics of Fluids, 33(3), 420 (1995).

9. Gleinser, W.; Braun, H.; Friedrich, C.; Cantow, J. - Polymer, 35(1), 128 (1994).

10. Macaúbas, P. H. P.; Demarquette, N. R. - Polymer, 42, 2543 (2001).

11. Lacroix, C.; Bousmina, M.; Carreau, P.J.; Favis, B. D.; Michel, A. - Polymer, 37(14), 2939 (1996).

12. Lacroix, C.; Aressy, M.; Carreau, P. J. - Rheologica Acta, 36, 416 (1997).

13. Huitric, J.; Médéric, P.; Moan, M.; Jarrin, J. - Polymer, 39 (20), 4849 (1998).

14. Mekhilef, N.; Carreau, P. J.; Favis, B. D.; Martin, F.; Ouhlal, A. - J Polym. Sci. Part B: Polymer Physics, 38,1359 (2000).

15. Carreau, P. J.; De Kee, D.; Chhabra, R. - Rheology of Polymeric Systems Principles and Applications; Carl Hanser Verlag, Munich Viena New York, (1997).

16. Underwood, E. E. - Quantitative Stereology, Addison Wesley, Reading, Massachusetts, (1970).

17. Lepers, J. C.; Favis, B.; Lacroix, C. J. - Polym. Sci., part B, Polymer Physics, 37, 939 (1999).

18. Wallheinke, K.; Pötschke, P.; Macosko, C.W.; Stutz, H. - Polym. Eng. Sci., 39 (6), 1022 (1999).
19. Yu, W; Zhou, C.; Inoue, T. - J. Polym. Sci., Part B, Polymer Physics, 38, 2378 (2000).

20. Yu, W; Zhou, C.; Inoue, T. - J. Polym. Sci., Part B, Polymer Physics, 38, 2390 (2000).

21. Baumgaertel, M.; Winter, H. H. - J. of Non-Newtonian Fluid Mechanics, 44, 15 (1992).

22. Honerkamp, J.; Wesse, J. - Rheologica Acta, 32, 65 (1993).

23. Winter, H.H.: J. of Non-Newtonian Fluid Mechanics, 68, 239 (1997).

24. Wu, S. - Polym. Eng. Sci., 27 (5), 335 (1987).

25. Wu, S. - "Polymer Interface and Adhesion", Marcell Dekker, Inc., New York, NY, (Chapter 3) (1982).

26. Arashiro, E. Y.; Demarquette, N. R. - J. Appl. Polym. Sci., 74, 1 (1999).

27. Demarquette, N.R. - "Tensão interfacial entre polímeros", São Paulo, 229 p, Tese de Livre Docência, Universidade de São Paulo (1999).

28. Rao, N.; Wanke, S. E.; FCIC; Sundararaj, U. - Can. Chem. News, april, 29 (1998).

29. Heino, M.; Kirjava, J.; Hietaoja, P.; Seppälä, J. - J. Appl. Polym. Sci., 1997, 65, 241.

30. Matos, M.; Favis, B. D.; Lomelline, P. - Polymer, 36, 3899 (1995).

31. Kim, H. C.; Nam, K. H.; Jo, W. H. - Polymer, 34 (19), 4043 (1993).

Recebido: $10 / 08 / 01$

Aprovado: 15/11/01 